\title{
THE TRANSLATRESS IN HER OWN PERSON SPEAKS: ESTUDIO DE LAS TRADUCCIONES DE APHRA BEHN A PARTIR DE LA TIPOLOGÍA DE DRYDEN
}

The translatress in her own person speaks: A study of Aphra Behn's translations based on Dryden's classification

Juan de Dios Torralbo Caballero*

Resumen

Este trabajo investiga las traducciones realizadas por Aphra Behn a partir de la clasificación tripartita que estableció John Dryden entre metáfrasis, paráfrasis e imitación. Behn cultiva la metáfrasis principalmente en sus traducciones de La Rochefoucauld, la paráfrasis en las versiones de Cowley o Tallemant, entre otros; mientras que aplica la imitación a las Fábulas de Esopo. Se constata que Behn rompe con la tradición de traducir principalmente a los autores clásicos, ensanchando los cauces de entrada de literatura moderna en Inglaterra y ampliando las posibilidades de la traducción literaria. Analizando unas muestras, se infiere la manipulación del texto original que la autora realiza en ocasiones, en consonancia con su ideario creativo, generando un texto meta provisto de nuevas resonancias de género y políticas contextualizadas en su época. Asimismo, se deduce que la escritora profesional de la Restauración cultiva la traducción movida, entre otros motivos, por los gustos del naciente mercado literario en cuanto a las novedades del país galo $\mathrm{y}$, por tanto, motivada por un afán comercial.

Palabras clave: Behn, traducción, Dryden, metáfrasis, paráfrasis, imitación.

Abstract

This paper examines the translations by Aphra Behn based on the tripartite classification that John Dryden established between metaphrase, paraphrase and imitation. Behn employs mainly metaphrase in her translations of La Rochefoucault; paraphrase in her versions of Cowley and Tallemant, among others; while applying the imitation to Aesop's Fables. It is found that Behn breaks with the tradition of translating principally classical authors, broadening channels for the entrance of modern literature into England and expanding the possibilities of literary translation. Analyzing the samples, the author's occasional manipulation of the original text is identified, consonant with her creative ideas and generating a metatext featuring new political and gender resonances contextualized in her era. It is also concluded that the professional writer of the Restoration employs translation spurred by the tastes of the burgeoning literary market, among other factors, shaped by recent developments in France and, as such, motivated by commercial interests

Key words: Behn, translation, Dryden, metaphrasis, paraphrasis, imitation. 


\section{INTRODUCCIÓN}

So thou by this translation dost advance / Our knowledge from the state of ignorance $^{1}$ (Behn, 1967, p. 167): estos dos versos confirman la buena estima que Aphra Behn (1640-1689) tiene acerca de la traducción literaria. Behn está alabando la traducción de Lucrecio realizada por Creech y, al mismo tiempo, está valorando el gran conocimiento que la traducción genera en el receptor, que en este caso es un lector femenino nominado de modo sinecdóquico mediante el adjetivo posesivo de plural (our). Aphra Behn (1994) considera el oficio de la traducción como una tarea digna, y la traductora lo explica en la dedicatoria de su obra Oroonoko (p. 3). Para Behn el poeta es como un pintor $^{2}$, que toma las ideas de la muestra pero las transforma porque se queda con la parte más noble, con el alma, con la mente:

My Lord, a picture drawer, when he intends to make a good picture, essays the face many ways, and in many lights, before he begins, that he may choose from the several turns of it which is most agreeable and gives it the best grace; [...] A poet is a painter in his way; he draws to the life, but in another kind; we draw the nobler part, the soul and mind; the pictures of the pen shall outlast those of the pencil, and even worlds themselves ${ }^{3}$.

Por otra parte, Behn relaciona la traducción no solamente con la creatividad, sino también con la innovación poética (Spearing, 2009, p. 154). Este artículo aplica la teoría de la traducción realizada por John Dryden a la poesía y a la obra de su autora contemporánea Aphra Behn. Asimismo, se pretende mostrar cómo la escritora moduló sus propios valores en muchas de sus traducciones incorporando contenido de carácter político e incluso personal y también contenido que podemos considerar femenino por medio de la manipulación pergeñada a los textos originales. Como hipótesis de trabajo mencionamos que Behn empleó diferentes formas de traducir a lo largo de su carrera literaria (Uman, 2012, p. 38).

\footnotetext{
${ }^{1}$ Se trata de los versos 41 y 42 del poema "To Mr. Creech (under the Name of Daphnis) on his Excellent Translation of Lucretius": "Con la traducción progresa / el saber de la ignorancia" (Trad. del autor).

${ }^{2}$ Es el tópico horaciano ut pictura poiesis que en la tradición insular postula Sir Philip Sidney (2002,p. 86) en su An Apology for Poetry, donde define a la poesía como 'a speaking picture'. Behn está asumiendo las premisas de la pintura para defender la dignidad de su oficio. Está reflexionando respecto de la naturaleza de su menester. Otro ejemplo del momento es el prólogo que escribió John Dryden (1695) para su traducción de Du Fresnoy (De Arte Graphica. The Art of Painting) titulado A parellel betwixt painting and poetry que en la editio princeps ocupa 56 páginas.

${ }^{3}$ Mi señor, un pintor de cuadros, cuando intenta realizar una buena pintura, ensaya con la cara varias veces, con muchas tonalidades. Antes de comenzar, de modo que pueda elegir entre diferentes modelos, el más aventajado y el de mayor gracia [...] Un poeta es como un pintor a su forma; dibuja desde la vida, pero de otra manera. Nosotros dibujamos la parte más noble, el alma y la mente. Los trazos de la pluma sobrevirirán a los del lápiz, e incluso a los planetas (Trad. del autor).
} 
La fuente más completa ${ }^{4}$ para consultar las obras de Aphra Behn es la editada por Janet Todd (1996) bajo el título general de The Works of Aphra Behn. La edición pionera de Montague Summers (1915) presenta algunos errores y está incompleta porque no contiene las máximas de La Rochefoucauld entre otras traducciones, ni el poema de Cowley o las Fábulas de Esopo (en el volumen sexto dedicado a la poesía). Germaine Greer (1989) recopila The Uncollected Verse of Aphra Behn donde también se echa en falta, por ejemplo, la metáfrasis del libro sexto de Cowley.

La metodología de trabajo empleada consiste, en primer lugar, en identificar los textos de Behn que son traducciones, o que están basados en textos originales de manera tangible; en segundo lugar, en clasificarlos mediante las tres categorías que conceptualizó Dryden realizando algunos estudios selectivos de los textos para corroborar dicha adscripción en una categoría u otra y plantear así una serie de conclusiones.

\section{LOS TRES TIPOS DE TRADUCCIÓN SEGÚN DRYDEN: METÁFRASIS, PARÁFRASIS E IMITACIÓN}

En 1680, John Dryden ${ }^{5}$ (1962, p. 268) concretamente en su prefacio a Ovid's Epistles, Translated by Several Hands teoriza su famosa tríada en torno a la actividad del traductor:

All translation, I suppose, may be reduced to these three heads. First, that of metaphrase, or turning an author word by word, and line by line, from one language into another. Thus [...] was Horace his Art of Poetry translated by Ben Jonson. The second was is that of paraphrase, or translation with latitude, where the author is kept in view by the translator, so as never to be lost, but his words are not so strictly followed as his sense, and that too is admitted to be amplified, but not altered. [...] The third way is that of imitation, where the translator (if now he has not lost that name) assumes the liberty not only to vary from the words and sense, but to forsake them both as he sees occasion $[\ldots]^{6}$.

Por tanto, la metáfrasis consiste en "turning an author word by word, and line by line, from one language to another" y se trata de la traducción ad pedem litterae.

\footnotetext{
${ }^{4}$ La edición al cuidado de Janet Todd fue publicada en la década de 1990. Actualmente Cambridge University Press está preparando otra edición completa (The Writings of Aphra Behn) al cuidado de las Dras. Elaine Hobby, Claire Bowditch, Mel Evans y Gillian Wright.

${ }^{5}$ El lector interesado en profundizar sobre los postulados de traducción en el siglo XVII puede consultar la obra de Muneharu Kitagaki, Principles and Problems of Translation in Seventeenth-century England, Kyoto, 1981.

${ }^{6}$ Toda traducción, mantengo, puede ser reducida a una de estas tres cabeceras. Primero, la metáfrasis, la aclimatación de un autor palabra por palabra, y verso a verso, de una lengua a la otra. Así [...] se aprecia en $E l$ arte de la poesía de Horacio traducido por Ben Jonson. El segundo es la paráfrasis, o la traducción con libertad, en la que la visión del autor es mantenida por el traductor, de modo que nunca se pierde, pero sus palabras no son seguidas tan estrictamente como su sentido, permitiéndose también ampliar, pero no alterar. [...] El tercer tipo es la imitación, donde el traductor (si es que no ha perdido su nombre) asume la libertad no solamente de variar las palabras y su sentido, sino también de prescindir de ellos cuando así lo considere oportuno [...]. (Trad. del autor)
} 
La paráfrasis es una traducción "with latitude where the author is kept in view by the translator, so as never to be lost, but his words are not so strictly followed as his sense; and that too is admitted to be amplified, but not altered" (Dryden, 1962, p. 268). Finalmente, la imitación contiene una total libertad pues en este caso el re-escritor provoca cambios en las palabras y en el sentido.

Si partimos de la premisa de que Behn ${ }^{7}$ era conocedora de la división que realizó Dryden acerca de la manera de traducir, la hipótesis se evidencia cuando comprobamos que Behn contribuyó a la colección drydeniana titulada Ovid's Epistles, particularmente con "A Paraphrase on Oenone to Paris, By Mrs. A. Behn". En su propia contribución, la escritora emplea el término 'paraphrase', que denota su conocimiento de las ideas preliminares escritas por Dryden.

En la época de Aphra Behn, en el siglo de Dryden, hallamos ejemplos de una y de otra. Ben Jonson compone un Art of Poetry que encaja en la definición de metáfrasis por la literalidad que presenta. La paráfrasis está cultivada en la traducción del libro cuarto que de la Eneida realizan Godolphin y Waller en tanto que el concepto de imitación cuadra bastante bien en la versión de Píndaro que realiza Abraham Cowley.

\section{LA METÁFRASIS DE BEHN EN EL CONTEXTO DEL SIGLO XVII: LA ROCHEFOUCAULD}

Este tipo de traducción es el que menos practica Behn, considerando su legado completo. No obstante se encuentran algunas muestras que siguen al pie de la letra la fuente del texto original. Se encuentra el mayor número de metáfrasis en las máximas de La Rochefoucauld que Behn tradujo.

Réflexions ou sentences et maximes morales se publica en 1665 en París. La traducción realizada por Behn está incluida en Reflections on Morality, or Seneca Unmasqued, concretamente en Miscellany, Being a Collection of Poems by Several Hands (Londres, 1685). En 1670 John Davis of Kidwelly traduce la obra como Epictetus Junior or Maxims of Modern Morality in Two Centuries, pero los estudios de Line Cottegnies (2004, p. 222) confirman que Aphra Behn no conoció esta versión inglesa. Hemos hallado otras traducciones al inglés pero de datación posterior, de la década de los 90, por lo que las versiones de Aphra son directas, a las que aplicaría cambios conscientemente.

Comencemos por la máxima 15 de La Rochefoucauld (2001, p. 5), "La clemence des princes n'est souvent qu'une politique pour gagner l'affection des peuples", que es traducida como "The Clemency of Princess is usually but a Policy to gain the Love of their Subjects" (Behn, 1996, p. 12), numerada como la máxima 4 en el texto meta. La única mutación que se aprecia es el cambio de la partícula negativa a la conjunción adversativa.

\footnotetext{
${ }^{7}$ Para un estudio respecto de los postulados teóricos que la escritora realizó en torno al oficio de la traducción, puede consultarse el trabajo pionero de Luis Pegenaute (1993, p. 145-156).
} 
Otra ilustración similar se observa en la traducción realizada a partir de la máxima 86 del texto original, "Notre défiance justifie la tromperie d'autruy" (La Rochefoucauld, 2001, p. 21), resultando en el texto meta como la máxima 51 de esta forma: "Our own mistrusts and Jealousies justifie the cheats of another" (Behn, 1996, p. 19).

Estas muestras son ejemplos de metáfrasis que Behn tradujo con total apego al texto original ${ }^{8}$. Se concluye que, como regla general, Behn realiza pocas metáfrasis, sin embargo se ha encontrado su mayor número en las traducciones de las máximas de La Rochefoucauld. Respecto de esta obra, Cottegnies (20047, p. 14) ha realizado un estudio estadístico concluyendo que el $43 \%$ de la obra de La Rochefoucauld que Behn traduce se puede considerar apegado al original y, por tanto -añadimos- se trata de metáfrasis.

La influencia de Seneca Unmasqued en la propia obra de Behn es tangible por varios aspectos. En primer lugar, el estilo desprovisto de ornato que cultiva en algunos de sus relatos revela la huella del estilo claro de La Rochefoucauld, según se aprecia en piezas como "The Wandering Beauty" (Behn, 1995b) o "The Unhappy Mistake" (Behn, 1995a). En segundo lugar, estos relatos -que fueron publicados póstumanente por Samuel Briscoe- contienen algunas aseveraciones en forma de máxima, como por ejemplo la que abre "The Unhappy Mistake", acerca de los celos, que dice así: "The Effects of Jelousie have even been more Fatal; and it is certainly one of the most Tormenting passions that an Humane Soul can be capable of, tho it be created by the least Appearances of Reason"9 (Behn, 1995a, p. 415), donde late la influencia de las máximas 2, 16, 51, 215, 320, 339, 359, 363, 370 y 371, según la numeración del texto de Behn, que tratan precisamente del asunto de los celos.

Para tener una idea cabal de la opinión crítica en torno a la traducción literaria en la Inglaterra de la segunda mitad del siglo XVII, conviene reflejar la discusión que John Denham ${ }^{10}$ realizó en 1656 a propósito de la traducción con el fin de comprobar que los literatos de la Restauración consideraron encomiable la creatividad aplicada por el traductor a partir de la obra original:

It is not business alone to translate Language into Language, but Poesie into Poesie; \& Poesie is of so subtile a spirit that in pouring out of one Language into another, it will all evaporate; and if a new spirit be not added in the transfusion, there will remain nothing but a Caput Mortuum ${ }^{11}$.

\footnotetext{
${ }^{8}$ Señalemos que según Margaret J. M. Ezell (2017, p. 354), Behn personaliza algunas máximas cambiando el género del hablante, así como se aprecia en la p. 338 a propósito de Lysander.

${ }^{9}$ Los efectos de los celos siempre han sido bastante fatales, y ciertamente se trata de las pasiones más tormentosas de las que el alma humana es capaz, aunque sean creados por la razón (Trad. del autor).

${ }^{10}$ Puede consultarse el texto de Sir John Denham (1779, p. 90-92), que insertó esta reflexión en el prefacio de The Destruction of Troy (1656, sin paginar), a partir del segundo libro de La Eneida deVirgilio.

${ }^{11}$ No consiste solamente en traducir de una lengua a otra, sino de la poesía a la poesía. La poesía posee un espíritu tan sutil que al verterla de una lengua a otra se evaporará; y si no se le infunden otros matices en la transfusión, no quedarán sino los restos (Trad. del autor).
} 
Otra aseveración data de ese mismo año, proveniente de Abraham Cowley $(1809$, p. 119) quien escribió en el prefacio a sus Pindaric Odes algunas de sus ideas en torno a la traducción e, igualmente, abogó por el significado general en detrimento de la copia literal del contenido:

If a man should undertake to translate Pindar word for word, it would be thought that one Mad man had translated another; [...] I have in these two Odesof Pindar taken, left out and added what I please; nor make it so much my aim to let the Reader know precisely what he spoke, as what was his way of manner and speaking $[\ldots]^{12}$.

\section{LA PARÁFRASIS: COWLEY Y TALLEMANT}

En la época de Aphra Behn la traducción de los clásicos se valoraba positivamente ${ }^{13}$, siendo cultivada por los escritores profesionales que ocuparon el panorama literario como es el ejemplo de John Dryden. Sin embargo, la autora que nos ocupa cierne su esfuerzo en Tallemant, La Rochefoucauld, Bonnecorse y Fontenelle, entre otros autores franceses coetáneos. Este hecho per se es una novedad que consideramos encuentra una de sus razones de ser en el potencial comercial que tienen para la traductora porque se trata de autores que en Francia estaban ocupando el naciente mercado literario. Aphra Behn traduce Le Voyage de l'Isle d'amour de Tallemant, unas máximas de La Rochefoucauld y la narración de Bonnecorse $L a$ Montre. Se trata de poesía alegórica y de prosa narrativa. En El Viaje a la Isla del Amor la recreadora cambia nombres propios y referencias contextuales para que el lector perciba un microcosmos coherente con el resto de la obra de la autora. Además, es una forma de complacer y de fidelizar al receptor.

Una traducción de la lengua latina llama la atención por tratarse su traductora de una mujer en el siglo XVII. En 1668 Abraham Cowley publicó Plantarum Libri Sex, compuesto de seis libros. En 1689, Nahum Tate editó la versión inglesa de la obra completa titulada Of Plants, aportando él mismo el prólogo así como las traducciones de los libros cuarto y quinto. A Behn le encargó la traducción del libro sexto que tituló "Of Trees" (1689) modulado en torno a 1726 versos (Todd, 1996, p. 409) y donde se acusa la presencia de Las Bucólicas de Virgilio (Laudien, 2006, p. 46). Paul Salzman (1994, p. 284) asevera que posiblemente Behn lo tradujo desde una versión inglesa y Luis Pegenaute (1993, p. 152) señala que "[e]s probable que Aphra Behn tuviera a mano una transliteración en prosa".

\footnotetext{
${ }^{12}$ Cuando un hombre se disponga a traducir a Píndaro palabra por palabra, podría pensarse que un loco habría traducido a otro; [...] En estas dos odas de Píndaro que he escogido, he omitido y añadido lo que me ha parecido oportuno; sin trasladar al lector exactamente lo que él dijo, sino más bien su forma y su lenguaje (Trad. del autor).

${ }^{13}$ Así se evidencia en los pronunciamientos que algunos escritores realizaron en la época, como el conde de Roscommon en su Essay on Translated Verse (1684), o en la alabanza dispensada por parte de Dryden en su quinta epístola.
} 
Si la poeta se queja en ocasiones de la falta de formación que tiene en lenguas clásicas, ahora demuestra su constancia y su interés al traducir unos textos que el famoso poeta contemporáneo Cowley ha compuesto en lengua latina. La escritora vierte el texto del último libro de Cowley partiendo de la plántula y fuente pero aplicando la amplificatio verborum. Según estudia Pegenaute (1993, p. 152), "N[ahum] Tate era amigo de la escritora y pudo haber sido él quien le sugiriera la idea de emprender esta transliteración en prosa".

Una mutación destacable es la operada en el verso 556 del texto original, cuando la autora traduce el sustantivo laurel, referido a los adornos que utilizan los reyes y los príncipes, y lo aplica metafóricamente al trofeo de la poesía, a la fama y a la posteridad. El texto de Cowley es: "[...] Dryas Immotis nemus undique ramis / Conticuit, vocemque arrectis frondibus hausit' (1668, p. 556-557). A nivel prosódico, se aprecia el cambio de metro a lo largo de todo el texto. Cowley (p. 143) genera su texto en hexámetros latinos y Behn lo escribe en 'heroic couplets':

And after the Monarchs, Poets claim a share

As the next worthy thy prized wreaths to wear.

Among that number, do not me disdain,

Me, the most humble of thy glorious Train.

Adjunta aparece una nota marginal indicando que "The Translatress in her own Person speaks" ${ }^{\text {"14 }}$ y junto con este apunte agrega los versos siguientes, a modo de coronación del poema, en los que reluce su gallarda proclamación como poeta, rogando no ser desdeñada; precisamente en unas líneas escritas en sus últimos días de vida (O’Donnell, 2004, p. 9). Behn se sitúa junto a Cowley (considerado como el árbol de laurel) y se regodea reclamando estar también junto al predicamento de Orinda, que es el nombre bucólico de la poeta Katherine Philips:

Among that number, do not me disdain,

Me, the most humble of that glorious Train.

I by a double right thy Bounties claim,

Both from my Sex, and in Apollo's Name:

Let me with Sappho and Orinda be

Oh ever sacred Nymph, adorn'd by thee;

And give my Verses Immortality.

Por un lado, se trata de una manipulación de la escritora, de una adición, ya que añade contenido que no consta en el original. De otra parte, manifiesta su deseo de fama, su corona de laurel, la búsqueda de la posteridad. Esta interpolación, al final del poema,

\footnotetext{
${ }^{14}$ La traductora habla por sí misma (Trad. del autor). Puede encontrarse esta referencia en el tomo primero de las obras completas editadas por Janet Todd, concretamente en la página 325 (The Works of Aphra Behn, ed. Janet Todd, Vol I. London: Pickering, 1992).
} 
significa la búsqueda de la inmortalidad y de la fama, según ha estudiado Sherry Simon (1996, p. 57). Es la representación de la escritora de modo consciente y claro.

El propio Dryden ${ }^{15}$ (1962, p. 273), al final de su prólogo a Ovid's Epistles, Translated by Several Hands, matiza que la escritora solamente acudió en una ocasión a la 'imitation'. El escritor profesional está alabando el esfuerzo de Behn al traducir desde las lenguas clásicas cuando escribió "I was desir'd to say that the Authour who is of the Fair Sex, understood not Latine. But if she does not, I am afraid she has given us occasion to be asham'd who do"16.

Esta traducción es relevante en el legado de Behn. De hecho, la poeta estimó que estaba excluida de las oportunidades que ofrece el conocimiento de las lenguas clásicas y lo lamentó en varios de sus trabajos ${ }^{17}$. Según estudia Elizabeth Spearing (2009, p. 175), para una mujer culta de su época, saber algo de latín y un poco de griego "would have been associated with access to the ideas and literature of classical paganism and with the possibility of escape from the strongly and perhaps stiflingly Christian framework of the vernacular writings most readily available to her" ${ }^{\text {"18 }}$. Este poema, sin embargo, es un ejemplo más del esfuerzo que Behn hizo. A esta traducción del latín hay que sumar su ya mencionada colaboración para el libro colectivo coordinado por Dryden, impreso por Jacob Tonson y titulado Ovid's Epistles.

Paul Tallemant publica en 1663 Le Voyage de l'Isle d'Amour in Recueil de quelques pièces nouvelles et galantes, el mismo año edita en Paris Le Voyage de l'Isle d'Amour, á Licidas. Las dos obras son traducidas por Aphra Behn. La primera está contenida en Poems upon Several Occasions, publicada en Londres en 1684 bajo el rótulo de $A$ Voyage to the Isle of Love. El segundo título también lo traduce Behn y lo publica cuatro años después (Londres, 1688) como Lycidus: or the Lover in Fashion, together with a Miscellany of New Poems. Además del mencionado potencial comercial de estas obras, consideramos que otro de los atractivos que sedujo a la traductora era su contenido acerca de la psicología del amor (Duffy, 1989, p. 238). Según coligió Janet Todd (1996, p. 321), Behn estaba fascinada con "the cartographies of love"19.

Behn "from the very beginning tends to add a sexual coloring to her source"20 (Spearing, 2009, p. 162) así como se aprecia en la descripción de la tormenta que encuentran los viajeros. El escritor francés escribe: 'Nour vogâmes paisiblement', 'un

\footnotetext{
${ }^{15}$ El trabajo de James Anderson Winn (1992) se puede consultar para tener una perspectiva completa de los elogios que Dryden escribió respecto del trabajo de Aphra Behn, particularmente las páginas 11-13.

${ }^{16}$ Yo deseaba afirmar que la autora, quien es mujer, no entendía latín. Pero si no lo entiende, me temo que nos ha dado a aquellos de nosotros que sí lo entendemos motivos para estar avergonzados (Trad. del autor)

${ }^{17}$ Un ejemplo es el poema que se ha nombrado en la nota primera. También lo lamenta en el prefacio a la comedia The Dutch Lover o en An Essay on Translated Prose.

${ }^{18}$ Podría haber estado vinculado con el acceso a las ideas y a la literatura del paganismo clásico y con la posibilidad de deshacerse de la férrea y dominante escritura vernácula de factura cristiana, accesible para ella con mayor facilidad (Trad. del autor).

${ }^{19}$ Las cartografías del amor (Trad. del autor).

${ }^{20}$ [Behn] desde el comienzo, tiende a agregar una nota sexual a su fuente (Trad. del autor).
} 
orage furieux', extendiéndose 8 líneas con 3 adjetivos y 2 adverbios respecto de la inclemencia meteorológica, sin referencias personales ni sentimientos. En cambio, Behn mantiene el sentido del original y lo amplía introduciendo la calma tras la tormenta $\mathrm{y}$, concretamente en los versos 74-116, genera una imagenería humana (donde no había vida). Behn "eroticizes the elemens in a most sensational manners"21 (Spearing, 2009, p. 163): "The ruffling Winds were hush'd in wanton sleep", "O'retoil-d and Panting, Calm and Breathless lay". Otro ejemplo es la intensificación de la tormenta en estos versos: "The billows all into disorder hurl'd / As if they meant to bury all the World".

En este sentido, Mirella Agorni (1998, p. 181) determinó el concepto de "'feminization' of early eighteenth-century literature on the activity of women translators" 22 , que consideramos de aplicación al modus operandi behniano aquí señalado. Otra ilustración, también demostrada por Spearing (2009, p. 163), es la amplificación de 6 versos en 23, añadiendo vitalidad y atributos llamativos al personaje femenino, enfatizando los atractivos sexuales de la mujer:

Car tout ce qu'a d'apas la brillante Jeunesse, Tout ci qui peut d'un coeur attirer la tendresse,

La fraicheur, l'Embon-point, la douce Majesté,

De la bouche $\&$ du teint la charmante beauté,

Des Roses \& des Lis le meslange agreable

Rendoient de ses beaux yeus le charme inevitable.

Such Charms of Youth, such Ravishment

Through all her Form appear'd, As if in her Creation Nature meant, She shou'd a-lone be ador'd and fear'd:

Her Eyes all sweet, and languishingly move, Yet so, as if with pity Beauty strove,

This to decline, and that to charm with Love.

A cheerful Modesty adorn'd her Face,

And bashful Blushes spread her smiling Cheeks;

Witty her Air; soft every Grace,

And 'tis eternal Musick when she speaks,

From which young listening Gods the Accents take

And when they wo'd a perfect Conquest make,

Teach their young favourite Lover so to speak.

[...]

\footnotetext{
${ }^{21}$ Erotiza los elementos de manera mejor (Trad. del autor).

${ }^{22}$ La 'feminización' de la literatura a comienzos del siglo XVIII en la actividad traductora de las mujeres (Trad. del autor).
} 
Se deduce que Behn aplica la estrategia de la amplificatio y de la manipulación del contenido que podemos definir como feminización, ya que Behn está interesada en el reflejo de una sensualidad que no está en la obra original.

Behn traduce otras obras francesas como La Montre (1666, París), de Balthazar de Bonnecorse, que resulta en La Montre: or the Lover's Watch ${ }^{23}$ o Les Entretiens sur la pluralité des mondes y Histoire des Oracles ${ }^{24}$ de Bernard Le Bovier de Fontenelle. Merece la pena indagar en las razones que motivaron a la escritora inglesa en la selección de este corpus. Una de ellas es su interés por la formación de la mujer en ciencias ${ }^{25}$ de la época. Según ha estudiado Violetta Trofimova (2007, p. 1) "Aphra Behn wanted women to give up caring about dresses and make-up and to study sciences and other useful things" "26 que explica las razones por las que Behn eligió las obras de Fontenelle, ya que pupularizó el "Copernican system for people who did not know Latin and did not have deep knowledge of Physics"27.

Se concluye que Behn realiza un buen número de paráfrasis en las traducciones de Cowley y Tallemant, entre otros $^{28}$, junto con algunas de La Rochefoucauld.

\section{LA IMITACIÓN: LAS FÁBULAS DE ESOPO}

En este apartado, además de las que seguidamente se estudian, hemos de incorporar el 40,5 de las traducciones de las máximas de La Rochefoucauld (Cottegnies, 2004, p. 14), pues han sido ya investigadas y conceptualizadas como adaptaciones libres hallándose en ocasiones incluso contradicciones con el texto de la obra fuente.

Las fábulas de Esopo se adscriben a la imitación según se analiza en este epígrafe. Estos relatos componen uno de los trabajos más amplios de Behn, que fue

\footnotetext{
${ }^{23}$ Behn la traduce 20 años después de la publicación original francesa.

${ }^{24}$ Los títulos ingleses de Behn son $A$ Discovery of New Worlds y History of Oracles. Hay que especificar que la obra de Fontenelle es una adaptación de otra obra holandesa escrita en latín por Van Dale.

${ }^{25}$ Behn está interesada en la epistemología de su tiempo, según muestra en trabajos como, por ejemplo, The Emperor of the Moon que contiene un eco de los conceptos heliocéntricos y de las teorías copernicanas, según ha estudiado recientemente Ha Inhye (2016, p. 423).

${ }^{26}$ Quería Aphra Behn que las mujeres dejaran de preocuparse por los vestidos y el maquillaje y que estudiaran ciencias y otras cosas de utilidad (Trad. del autor).

${ }^{27}$ El sistema copernicano para personas que no sabían latín ni tenían un conocimiento profundo de física (Trad. del autor).

${ }^{28}$ Las versiones de La Rochefoucauld también deben citarse en este apartado de paráfrasis. Cottegnies (14) deduce que el $16,5 \%$ de las traducciones de las máximas son paráfrasis. Destaca un tipo de cambio en el texto meta que está relacionado con la defensa de la mujer que la autora realiza. La traducción 143 ("It appears that Nature has prescribed every Body from their Birth limits for their Virtues and their Vices") (Behn, 1996, p. 30), basada en la máxima original 189 ("Il semble que la nature ait prescrit à chaque homme dés sa naissance des bornes pour les vertus \& pour les vices") (La Rochefoucauld, 2001, p. 49), muestra cómo la poeta inglesa neutraliza las referencias masculinas desproveyendo el texto del usual sesgo androcéntrico de su época. Esta deducción se relaciona con la conclusión mencionada de Margaret J. M. Ezell (2017, p. 354).
} 
publicado en 1687 por Francis Barlow. La poeta inglesa escribe 110 traducciones. Behn recreó las fábulas como piezas de acompañamiento a los grabados de Francis Barlow, traduciendo también 31 comentarios de dichas ilustraciones, en una edición que contenía versiones en inglés, en francés y en latín.

Hay que mencionar, sin embargo, que en 1666 existía una versión que ya incorporaba los textos en versión trilingüe. El autor de los textos en latín y en francés fue Robert Codrington, mientras que las versiones inglesas eran autoría de Thomas Philipott. La nueva edición de 1687 (en la que participó Behn) presentaba una visible novedad, que los textos de Philipott fueron cambiados por los de la escritora, cuyas traducciones eran más breves. Mientras que las versiones de Philipott son más abstractas, las de Behn son más claras, y mientras que el estilo del primero es más opaco con tendencia a la desorganización sintáctica, las versiones de Behn no presentan esa tendencia al hipérbaton. En este sentido, podemos deducir que la mayor precisión en la escritura inglesa que genera Behn se relaciona con la claridad de carácter casi periodístico (Torralbo, 2013, p. 70-76) que cultivan los poetas de su época como es el caso de John Dryden. La versión que Behn ${ }^{29}$ presenta de las fábulas es, por tanto, contextual y considera a los lectores-receptores de su obra. Las composiciones de Behn son textos actualizados y modernizados, con referencias a la sociedad y a la política del momento.

Aphra Behn injerta referencias al contexto político de sus días, como se aprecia en "The Ringdove and Fowler" cuya frescura temática recuerda el intento de usurpar el trono a Jacobo II y pone de manifiesto la decantación de la poeta por la naciente facción tory. Se entrevé su preferencia por el bando católico de La Casa Reall ${ }^{30}$ de Estuardo:

While the young fowler sought, with eager skill,

A Ring Dove in a neighbouring Tree to kill.

And Adder in a floury mead beneath,

Stung the unhappy youth, and stung to death.

Moral

The young usurper, who design'd t'invade

And others right, himselfe the victim made (Aesop, 1687, p. 25).

En este poema no habla directamente la torcaz ni tampoco el cazador y la moraleja enclaustra una referencia clara para el lector de la época, la conspiración del protestante James Scott, duque de Monmouth, quien capitanea una treta para ocupar el trono de Jacobo II. La moraleja que aparece en la versión latina del texto original ("De Aucipe \& Palumbe") es: "Monemur hac Fabella cum consideratis ambulare,

\footnotetext{
${ }^{29}$ Los textos se citan desde la edición de 1687, Aesop's Fables with his Life: In English, French and Latin. Newly translated [...]. London: Printed for Francis Barlow.

${ }^{30}$ La antología comentada de Andrew McRae y John West (2017, p. 259) o el estudio de Margaret J. M. Ezell (2017, p. 321) así lo corroboran.
} 
saepissime etenim videmus eos circumveniri, qui res novas moliuntur" (Aesop, 1687, p. 25). Por tanto, los cambios que aplica la traductora son didácticos y están ligados al entramado sociopolítico de sus días, como se aprecia en "The Wood and Clown" ("Rusticus \& Sylva"), donde se refiere a los ciudadanos que se rebelan contra sus príncipes si estos les dan muchas libertades. La moraleja es: "Ungratefull People this on princes fall, / And given some liberties rebell for all", mientras que el texto latino reza "Quod levissima \& quae minutuli videntur Momenti, in gravissimum nonnunquam tendunt dispendium" (p. 197).

"The City Mouse and Country Mouse" es otra fábula en la que deseamos detenernos, para señalar que la traductora cambia la prosa de Esopo por el verso en inglés, resultando en un cuarteto que prepara el terreno para la moraleja articulada en un pareado de pentámetros yámbicos:

The City Mouse invites her Country Guest,

To tast the daintyes of a City feast,

But oft disturb'd by interrupted noys,

They hide, and fear his appetite destroys.

Moral

The great the Hurry of the world indure,

And tis the country life alone's secure (p. 35).

El texto latino, "De Mure Urbano \& Mure Rustico", comienza así: "Mus Rusticus videns Urbanum Murem Rus deambulantem invitat ad caenam, depronitque ommne penu, ut tanti hospitis expleat lautitiam" (Aesop, 1967, p. 35). Según se comprueba con este ejemplo, el texto meta queda abreviado yendo a la quintaesencia del asunto, que aparece levemente mutada. Una suma notable es la inserción del tema del beatus ille que planta Aphra Behn en su poema. El texto origen se centra en la comida opípara, llena de peligros, que tiene a mano el ratón de ciudad. Este ratón urbano desdeña la frugalidad y la dificultad que abraza el ratón de campo para subsistir. Aquí surge el contraste entre la opulencia y la humildad del feliz ratón campesino, el que una vez prueba la vida del casero prefiere no dejar sus terrones al aire libre, bajo los que busca y encuentra su comida sin necesidad de pasar miedo robando. A pesar de la transformación emanada al final de la fábula en inglés (el texto latino es "Divitiae quidem prae se ferunt voluptatem, sed, si penitus introspicias, habent pericula et amaritudinem. Maiori etenim curarum mole opprimuntur divites, quam pauperes") (p. 35), el poema de Behn es ágil y claro mostrando la clave del texto esópico así como la enjundia del devenir argumental del mismo.

Con las fábulas de Esopo la escritora se inserta en la amplia tradición europea que iniciaran Alexander Neckam, Walter of England, Rumi, Odo of Cheriton, John of Sheppey y continuara William Caxton (The Fables of Aesop, 1484) y, de modo más cercano, Roger L'Estrange (Fables of Aesop, 1692, 1699) o Jean de La Fontaine. Dentro de la tradición fabulística insular también hay que citar a Anne Finch ("The 
Nightingale and the Cuckoo", "The Critick and the Writer of Fables" (c. 1688-1717)) y al mismo John Dryden (The Hind and the Panther, 1687; "The Cock and the Fox", 1700). Luego seguirán brotando en manos, por ejemplo, de Christopher Smart (1765, Poetical Translation of the Fables of Phaedrus). Se trata de una estela que arranca en las traducciones latinas del poeta romano Fedro de quien han sobrevivido más de un centenar de fábulas. Igual que Behn, Fedro adapta el mensaje de sus versos a la sociedad del momento. Las fábulas de Esopo trascienden, por tanto, el objetivo filosófico y moral engendrado en el texto origen.

La manipulación del texto fuente no es nueva. El romano Brabio vierte al griego la colección de fábulas que nos ocupa aplicando brevedad y humor a su trabajo. Rómulo, por su parte, ofrece unas paráfrasis en prosa. El clérigo inglés Odo of Cheriton es el responsable de la traducción de las fábulas a latín medieval y es entonces cuando toman un significado cristiano. De hecho, este predicador católico ofrece sus piezas acompañadas de un sermón ilustrativo del nuevo significado alegórico que ha conferido a cada fábula. Esta versión destaca también por su misoginia que luego combatirá Marie de France. Tal como adujo Lee Rourke (2011, p. 40) en su estudio, las fábulas donan una tradición antropomórfica que permanece perenne en la historia literaria desde esos momentos.

La labor de Aphra Behn es un claro ejemplo de manipulación del texto original, es una muestra de cómo la literatura, concretamente la traducción literaria, presenta mutaciones en función de la ideología del nuevo escritor. Behn pone su literatura al servicio de la monarquía.

Behn vierte la prosa en poesía y mantiene una destacable simetría y regularidad en sus versiones, porque constan de un cuarteto modulado mediante pentámetros yámbicos. Cada fábula está coronada por una moraleja que cierra la composición mediante un pareado, también compuesto en pentámetros yámbicos. Balbina Prior (2004, p. 37) señala que los versos de Behn presentan una singularidad que no era frecuente en su época, que es la terminación en preposiciones, artículos o determinantes.

El tercer tipo que conceptualizó Dryden, la imitación, se evidencia principalmente en Las fábulas de Esopo junto con otros poemas que pueden adscribirse a esta categoría. Sirva como botón de muestra el caso de "Disappointment", que bebe de las fuentes de "L'Occasion perdue recouverte", del autor francés Jean Benech de Catenac. Concretamente los versos 8-10 del francés y los versos 9-10 del inglés son así:

cet ennemy de Cypris

Ne laissoit de lumiere au monde

Que dans les beaux yeux de Cloris.

And left no Light to guide the World, But what from Cloris Brighter Eyes was hurld 
Con esta muestra se comprende cómo cambian los roles semánticos. Lo más destacable es que Cloris no aparece como objeto pasivo del deseo sexual de Lisandro $^{31}$. Cloris emerge en la nueva creación como un sujeto activo. Como norma general, a la mujer se le representa como receptáculo pasivo de las apetencias del varón (Goreau, 1980, p. 183), modelándolas como objeto del deseo masculino. En la época de la Restauración, la poesía de amor alberga la batalla entre hombres y mujeres.

Colegimos, coincidiendo con Uman (2012, p. 38), que como regla general Behn muestra una mayor fidelidad al texto fuente en sus traducciones de prosa, mientras que en sus versiones de poesía aplica una notoria libertad a la hora de verter el contenido y la forma del texto original en el texto meta.

\section{CONCLUSIÓN}

Aphra Behn inaugura un nuevo paradigma en la traducción literaria y ensancha sus cauces de difusión. Los poetas del siglo XVII siguen los modelos mayoritariamente cultivados con anterioridad en Inglaterra, a saber, tornan la mirada hacia el pasado grecolatino como hacen Carew, Donne, Oldham o Dryden. Según se investiga en este trabajo, Aphra Behn rompe la tradición y ensancha el elenco de posibilidades de la traducción. Las traducciones de Aphra Behn provienen, en su mayoría, de autores franceses contemporáneos, lo que denota el conocimiento de la lengua gala que tuvo la escritora.

Ovid's Epistles, Translated by Several Hands se trata de una obra coordinada por John Dryden y publicada en 1680. Para este artículo es fundamental mencionar este trabajo colectivo en el que participaron 16 escritores. Según señala Irwin Primer (2011, p. 159), es destacable que Aphra Behn sea la única mujer que participó en esta edición.

Otros escritores de la época defendieron la paráfrasis y escribieron sus postulados en contra de la metáfrasis por considerarla como 'servile'. Sir John Denham $(1779$, p. 90$)$ considera, en $1656^{32}$ que "it is not his business alone to translate Language into Language, but Poesie into Poesie [...] that in pouring out of one Language into another, it will all evaporate; that if a new spirit be not added in the transfusion, there will remain but a Caput mortuum [...]"33.

Las tres características más sobresalientes que Behn aplica en sus traducciones son la amplificatio verborum, la feminización y la politización, plasmando de este modo unos textos meta al trasluz de su ideario estético y social, generando más versos cuando ella lo considera necesario.

${ }^{31}$ Resulta ilustrativo comparar este poema con la versión de John Wilmot, Earl of Rochester (2004, p. 15), “The Imperfect Enjoyment” y con la de George Etherege, donde la semántica cambia respecto de la planteada por Aphra Behn.

${ }^{32}$ Este postulado se encuentra en el prefacio a The Destruction of Troy.

${ }^{33}$ Véase la traducción reflejada en la nota 11. 
Si John Dryden es el hombre de letras del siglo XVII, Aphra Behn es la mujer de letras de su tiempo; porque tanto con Dryden como con Behn podemos hablar de poetas profesionales. Aphra Behn (1994, p. 3) tiene el sentido de la permanencia de la obra de arte verbal; pues explica que los dibujos de la pluma sobrevivirán a los del pincel y perdurarán con el tiempo. Por muy humildes que sean los frutos de su trabajo, según nos dice en reiteradas ocasiones ("such humble fruits as my industry produces" ${ }^{\text {"34; }}$ Behn, 1994, p. 5), en sus traducciones hay un plan y un proyecto que va más allá de la tradición y que busca un lugar destacado entre los escritores de su tiempo.

La poeta busca el sostenimiento económico, de ahí los nuevos modelos, de ahí la selección del corpus a traducir. Es la conciencia creadora que se funde en la difusión impresa de quien también había frecuentado el tablado teatral de pane lucrando. Quiere que la literatura llegue al público, que el público (el vulgo lopista) se interese y la compre. La potestas que hubiera supuesto seleccionar en todo momento patrones clásicos deja paso a la auctoritas que genera auténticas reescrituras ensanchando de este modo los cauces literarios de la época.

\author{
Universidad de Córdoba* \\ Facultad de Filosofia y Letras \\ Departamento de Filología Inglesa y Alemana \\ Plaza del Cardenal Salazar, 3, Córdoba (España) \\ torralbocaballero@uco.es
}

\title{
OBRAS CITADAS
}

Aesop, Aphra Behn, Francis Barlow, Thomas Philipot and Robert Codrington. (1687). Aesop's Fables with his life: in English, French and Latin. Newly translated [...]. London: Printed by H. Hills jun. for Francis Barlow.

Agorni, Mirella. (1998). "The Voice of the 'Translatress': From Aphra Behn to Elizabeth Carter". The Yearbook of English Studies, 28, 181-195.

Behn, Aphra. (1996). Seneca Unmasqued and Other Prose Translations. The Works of Aphra Behn. Vol 4. Ed. Janet Todd. Columbus: Ohio State University Press.

— (1995a). "The Unhappy Mistake; or, The Impious Vow Punished." The Works of Aphra Behn. The Fair Jilt and Other Short Stories. Vol III. Ed. Janet Todd. London: William Pickering, 411-442.

(1995b) "The Wandering Beauty." The Works of Aphra Behn. The Fair Jilt and Other Short Stories. Vol III. Ed. Janet Todd. London: William Pickering, 388-409. (1994). Oronooko, or the Royal Slave. London: Oxford University Press.

Montague Summers (Ed.). (1915). The Works of Aphra Behn, Vol. VI. New York: Bejamin Blom.

\footnotetext{
${ }^{34}$ Esos frutos humildes que produce mi esfuerzo (Trad. del autor).
} 
Cottegnies, Line. (2004). "Aphra Behn's French Translations". The Cambridge Companion to Aphra Behn. Eds. Hughes, Derek and Janet Todd. Cambridge: Cambridge University Press, 221-234.

Cowley, Abraham. (1809). "Preface". The Works in Prose and Verse of Mr. A. Cowley; with Notes by Dr. Hurt. London: Printed for John Sharpe, 119-122.

— (1668). The Works of Mr Abraham Cowley. [...] The Second and Third Parts. London: J M for Henry Herringman / Charles Harper, [1700].

De la Rochefoucauld, Francoise. (2001). Seneca Unmasqued. A Bilingual Edition of Aphra Behn's Translation of La Rochefoucauld's Maximes. Ed. Irwin Primer. New York: AMS Press.

Denham, John. (1779). "Preface to The Destruction of Troy". The Poetical Works $f$ Sir John Denham. With the Life of the Author. Edinburg: Apollo Press, 89-92.

Dryden, John. (1695). De arte graphica. The art of painting by C. A. Du Fresnoy with Remarks. London: Printed by J. Heptinstall for W. Rogers.

— (1962). Of Dramatic Poesy and Other Critical Essays. Vol I. Ed. George Watson. London: J. M. Dent \& Sons.

Duffy, Maureen. (1989). The Passionate Shepherdess. The Life of Aphra Behn 16401689. London: Phoenix Press.

Ezell, Margaret, J. M. (2017). The Oxford English Literary History: Volume 5: 1645 1714: The Later Seventeenth Century. Oxford: Oxford University Press.

Goreau, Angeline. (1980). Reconstructing Aphra. A Social Biography of Aphra Behn. New York, The Dial Press.

Greer Germaine (Ed.). (1989). The Uncollected Verse of Aphra Behn. Saffron Walden, Essex: Stump Cross Books.

Inhye Ha. (2016). "Cosmic Blindness, Comic Blindness: The Lunar Imagination and Women's Place in Aphra Behn's Emperor of the Moon". The Journal of English Language and Literature 62, 423-440.

Kitagaki, Muneharu. (1981). Principles and Problems of Translation in Seventeenthcentury England. Kyoto: Yamaguchi Schoten.

Laudien, Heidi. (2006). "Ladies of the Shade: The Pastoral Poetry of Aphra Behn and Elizabeth Singer Rowe". The Female Wits. Women and Gender in Restoration Literature and Culture. Eds. Pilar Cuder-Domínguez, Zenón Luis-Martínez and Juan A. Prieto-Pablos. Huelva: Servicio de Publicaciones de la Universidad de Huelva, 43-63.

McRae, Andrew and John West. (2017). Literature of the Stuart Successions. An Anthology. Manchester: Manchester University Press.

O'Donnell, Mary Ann. (2004). "The Documentary record". The Cambridge Companion to Aphra Behn. Ed. Janet Todd. Cambridge: Cambridge University Press, 1-11.

Pegenaute Rodríguez, Luis. (1993). “Aphra Behn (1640-89): Traductora y teórica de la traducción”. Livius: Revista de estudios de traducción 4, 145-156. 
Primer, Irwin. (2001). "Notes". Seneca Unmasqued. A Bilingual Edition of Aphra Behn's Translation of La Rochefoucauld's Maximes. New York: AMS Press, 153-176.

Prior, Balbina. (2004). “Aphra Behn: Espía universal contra toda esclavitud”. Las fábulas del deseo y otros poemas. Sial: Madrid, 9-56.

Rourke, Lee. (2011). A Brief History of Fables. From Aesop to Flash Fiction. London: Hesperus Press Limited.

Salzman, Paul. (1994). "Introduction and notes". Aphra Behn, Oroonoko and other Writings. Oxford: Oxford University Press.

Sidney, Sir Philip. (2002). An Apology for Poetry. Ed. R. W. Maslen. Manchester: Manchester University Press.

Simon, Sherry. (1996). Gender in Translation. Cultural Identity and the Politics of Transmission. London: Routledge.

Spearing, Elizabeth. (2009). "Aphra Behn: the politics of translation". Aphra Behn Studies. Ed. Janet Todd. Cambridge: Cambridge University Press, 154-177.

Todd, Janet. (1996). The Secret Life of Aphra Behn. London: Andre Deutsch.

Torralbo Caballero, Juan de Dios. (2013). Una nueva poesía en la literatura inglesa: Dryden y Pope. Sevilla: Alfar.

Trofimova, Violetta. (2007). "French influence on English culture in the second part of the seventeenth century: Aphra Behn as a creative translator and a Mediator between the two cultures". Culture, language and representation: Cultural Studies Journal of Universitat Jaume I 4, 241-255.

Uman, Deborah. (2012). Women as Translators in Early Modern England. Newark: University of Delaware Press.

Wilmot, John. (2004). Earl of Rochester. Selected Works. Ed. Frank H. Ellis. London: Penguin.

Winn, James Anderson. (1992). 'When Beauty Fires The Blood': Love And The Arts In The Age Of Dryden. Ann Arbor: University of Michigan Press. 